\title{
ON THE EXISTENCE OF NORMAL SQUARE AND NTH ROOTS OF OPERATORS
}

\author{
MOHAMMED HICHEM MORTAD
}

\begin{abstract}
The primary purpose of this paper is to show the existence of normal square and nth roots of some classes of bounded operators on Hilbert spaces. Two interesting simple results hold. Namely, under simple conditions we show that if any operator $T$ is such that $T^{2}=0$, then this implies that $T$ is normal and so $T=0$. Also, we will see when the square root of an arbitrary bounded operator is normal.
\end{abstract}

\section{INTRODUCTION}

Let $H$ be a complex Hilbert space, and let $B(H)$ denote the algebra of all bounded linear operators on $H$.

By a square root of $A \in B(H)$, we mean a $B \in B(H)$ such that $B^{2}=A$. An $A \in B(H)$ is called positive if

$$
<A x, x>\geq 0, \forall x \in H .
$$

It is known that any positive operator $A$ has a unique positive square root (which is denoted by $\sqrt{A}$ or $A^{\frac{1}{2}}$ and these notations are exclusively reserved to the unique positive square root). However, there are non normal $N$ such that $N^{2}$ is normal. As an extreme example, just consider any non-normal $N$ such that $N^{2}=0$. In fact, the identity $2 \times 2$ matrix $I$ has an infinite number of self-adjoint square roots. Indeed, the self-adjoint

$$
A_{x}=\left(\begin{array}{cc}
x & \sqrt{1-x^{2}} \\
\sqrt{1-x^{2}} & -x
\end{array}\right)
$$

represents a square root of $I$ for each $x \in[-1,1]$.

There are some quite known research papers on this topic. For instance, readers may wish to consult: [2], [4], [7], [8], [9] and [12]. In particular, Putnam [7] gave a condition guaranteeing that the square root of a normal operator be normal. The problem considered in this paper is of this sort.

Recall that any $T \in B(H)$ is expressible as $T=A+i B$ where $A, B \in B(H)$ are self-adjoint. As it is customary, we denote $A$ by $\operatorname{Re} T$ and $B$ by $\operatorname{Im} T$.

It is also known (cf. [6]) that $T=0$ iff $A=B=0$. Therefore, if $A+i B=C+i D$, then $A=C$ and $B=D$ whenever $A, B, C, D$ are self-adjoint. It is also easily verifiable that $T$ is normal iff $A B=B A$.

We also recall some known results which will be called on below (these are standard facts, see [6] for proofs).

2010 Mathematics Subject Classification. Primary 47A62, Secondary 47A05.

Key words and phrases. Square roots. Normal, self-adjoint and positive operators. Real and imaginary parts of an operator. Spectrum. Cartesian decomposition. 
Theorem 1.1. Let $A, B \in B(H)$ be self-adjoint. Then:

$$
0 \leq A \leq B \Longrightarrow \sqrt{A} \leq \sqrt{B}
$$

Proposition 1.2. Let $A, B \in B(H)$ be self-adjoint. Then

$$
|A| \leq B \Longrightarrow-B \leq A \leq B,
$$

where $|A|=\sqrt{A^{*} A}$.

In the end, we assume that readers are familiar with other notions and results on $B(H)$.

\section{Existence of Normal Roots}

Theorem 2.1. Let $C \in B(H)$ be self-adjoint and let $T=A+i B \in B(H)$ be such that $T^{2}=C$.

(1) If $\sigma(A) \cap \sigma(-A)=\varnothing$, then $T$ is self-adjoint and invertible.

(2) If $\sigma(B) \cap \sigma(-B)=\varnothing$, then $T$ is skew symmetric (that is, $T^{*}=-T$ ) and invertible.

The proof is based upon the following known result:

Lemma 2.2. (10] or [1]) Let $A, B \in B(H)$ be such that $\sigma(A) \cap \sigma(B)=\varnothing$. Then the equation $A X-X B=S$ has a unique solution $X$ (in $B(H)$ ) for each $S \in B(H)$.

Now, we prove Theorem 2.1:

Proof. We have

$$
T^{2}=C \Longleftrightarrow T^{2}=A^{2}-B^{2}+i(A B+B A)=C .
$$

By the self-adjointness of $A$ and $B$, we obtain the self-adjointness of $A^{2}-B^{2}$ and $A B+B A$ as well. Hence

$$
\left\{\begin{array}{c}
A^{2}-B^{2}=C, \\
A B+B A=0 .
\end{array}\right.
$$

(1) If $\sigma(A) \cap \sigma(-A)=\varnothing$, then Lemma 2.2 says that the equation

$$
A B-B(-A)=A B+B A=0
$$

has a unique solution which is necessarily $B=0$. Hence $T=A$ is selfadjoint. If $0 \in \sigma(T)$, then $0 \in \sigma(A)$ and so $0 \in \sigma(-A)$ too. This, however, would violate the assumption $\sigma(A) \cap \sigma(-A)=\varnothing$. Therefore, $T$ is invertible.

(2) When $\sigma(B) \cap \sigma(-B)=\varnothing$, a similar method yields $A=0$, i.e. $T=i B$. Hence $T$ is invertible because $\sigma(B) \cap \sigma(-B)=\varnothing$.

A result by Embry [3] may be readjusted as follows:

Lemma 2.3. Let $A, B \in B(H)$ be normal and such that $A B=-B A$. Designate the numerical range of $A$ by $W(A)$. If $\sigma(A) \cap \sigma(-A)=\varnothing$ or $0 \notin W(A)$ (resp. $\sigma(B) \cap \sigma(-B)=\varnothing$ or $0 \notin W(B)$ ), then $B=0$ (resp. $A=0)$.

Using the same method as above and the foregoing lemma, we may establish:

Proposition 2.4. Let $C \in B(H)$ be self-adjoint and let $T=A+i B \in B(H)$ be such that $T^{2}=C$. If $0 \notin W(A)$ (or $0 \notin W(B)$ ), then $T$ is self-adjoint. As above, in the first case $T=A$ and in the second $T=i B$. 
We know that if $T \in B(H)$, then $T^{2}=0$ does not, in general, imply that $T=0$. We also know that if $T$ satisfies $\left\|T^{2}\right\|=\|T\|^{2}$ (for example, if $T$ is self-adjoint or normal or normaloid in general i.e. $\left\|T^{n}\right\|=\|T\|^{n}$ for all $n$ ), then $T^{2}=0$ does imply that $T=0$. The following result is therefore of interest.

Proposition 2.5. Let $T \in B(H)$ be such that $T^{2}=0$. If $\operatorname{Re} T \geq 0$ (or $\operatorname{Im} T \geq 0$ ), then $T$ is normal and so $T=0$.

Proof. Write $T=A+i B$ where $A, B \in B(H)$ are self-adjoint where $A=\operatorname{Re} T$ and $B=\operatorname{Im} T$. Then clearly

$$
T^{2}=A^{2}-B^{2}+i(A B+B A)
$$

So, if $T^{2}=0$, then

$$
A^{2}-B^{2}+i(A B+B A)=0 \Longrightarrow\left\{\begin{array}{c}
A^{2}=B^{2} \\
A B=-B A
\end{array}\right.
$$

Hence, if $A \geq 0$ (a similar argument works when $B \geq 0$ ), then

$$
A B=-B A \Longrightarrow A^{2} B=-A B A=B A^{2} \Longrightarrow A B=B A .
$$

Therefore, $T$ is normal. Accordingly,

$$
\|T\|^{2}=\left\|T^{2}\right\|=0 \Longrightarrow T=0,
$$

as suggested.

Corollary 2.6. Let $T \in B(H)$ be such that $T^{2}=0$. If any of $\sigma(\operatorname{Re} T)$ or $\sigma(\operatorname{Im} T)$ is a subset of either $\mathbb{R}^{+}$or $\mathbb{R}^{-}$, then $T$ is normal and so $T=0$.

Remark. The condition $\operatorname{Re} T \geq 0$ (or $\operatorname{Im} T \geq 0$ ) in Proposition 2.5) is, in general, not sufficient to make $T$ normaloid. Indeed, if $V$ is the Volterra operator on $L^{2}[0,1]$ say, then (cf. [6]) $V$ is not normaloid as

$$
r(V)=0 \neq \frac{2}{\pi}=\|V\|
$$

where $r(V)$ denotes the spectral radius of $V$. It can, however, easily be checked that $\operatorname{Re} V \geq 0$.

We finish this section with a general criterion guaranteeing the normality of the square root (this generalizes Proposition 2.5):

Theorem 2.7. Let $S=C+i D \in B(H)$ be such that $T^{2}=S$ where $T=A+i B \in$ $B(H)$. Then (if $[\cdot, \cdot \cdot]$ denotes the usual commutator)

$$
[B, C]=[A, D]
$$

and

$$
[A, C]=[B, D]
$$

(Consequently, $B C=C B \Leftrightarrow A D=D A$ and $A C=C A \Leftrightarrow B D=D B$ ).

If $A \geq 0$ (or $A \leq 0$ ), then

$$
T \text { is normal } \Longleftrightarrow A D=D A \text {. }
$$

If $B \geq 0$ (or $B \leq 0)$, then

$$
T \text { is normal } \Longleftrightarrow B D=D B \text {. }
$$

In particular, if $S$ is self-adjoint (i.e. $D=0$ ) and $A \geq 0$ or $A \leq 0$ or $B \geq 0$ or $B \leq 0$, then $T$ is always normal. 
Proof. By assumption,

$$
A^{2}-B^{2}+i(A B+B A)=C+i D \Longrightarrow\left\{\begin{array}{c}
A^{2}-B^{2}=C \\
A B+B A=D .
\end{array}\right.
$$

Hence

$$
A B+B A=D \Longrightarrow A^{2} B+A B A=A D \text { and } A B A+B A^{2}=D A
$$

and so

$$
A^{2} B-B A^{2}=A D-D A .
$$

Since $A^{2}=B^{2}+C$, we get

$$
B C-C B=A D-D A .
$$

Also,

$$
A B+B A=D \Longrightarrow A B^{2}+B A B=D B \text { and } B A B+B^{2} A=B D
$$

and so as above

$$
B^{2} A-A B^{2}=B D-D B
$$

and by invoking $B^{2}=A^{2}-C$, we obtain

$$
A C-C A=B D-D B .
$$

To show the last two assertions, we have from above that

$$
A^{2} B=B A^{2} \Longleftrightarrow A D=D A \text { and } B^{2} A=A B^{2} \Longleftrightarrow B D=D B .
$$

So if $A \geq 0$, then clearly

$$
A^{2} B=B A^{2} \Longleftrightarrow A B=B A,
$$

and the previous holds iff $T=A+i B$ is normal. A similar reasoning applies when $A \leq 0$. Finally, argue similarly in the event $B \geq 0$ or $B \leq 0$ and this completes the proof.

\section{Explicit Construction of Roots}

It is known (cf. [11]) that if $N$ is normal with a spectral integral $\int_{\sigma(N)} \lambda d E$ where $E$ is a spectral measure, then $\int_{\sigma(N)} \sqrt{\lambda} d E$ is square root of $N$ where $\sqrt{\lambda}$ is a complex square root of $\lambda$.

Under an extra condition on the normal operator, we can have an even more explicit formula.

Theorem 3.1. Let $N=C+i D \in B(H)$ be normal with either $D \geq 0$ or $D \leq 0$ (equivalently, $\sigma(D) \subset \mathbb{R}^{+}$or $\sigma(D) \subset \mathbb{R}^{-}$). When $D \geq 0$, then

$$
T=\left(\frac{|N|+C}{2}\right)^{\frac{1}{2}}+i\left(\frac{|N|-C}{2}\right)^{\frac{1}{2}}
$$

is a normal square root of $N$. If $D \leq 0$, then

$$
T=\left(\frac{|N|+C}{2}\right)^{\frac{1}{2}}-i\left(\frac{|N|-C}{2}\right)^{\frac{1}{2}}
$$

is another normal square root of $N$. 
Proof. The hardest part of the proof is the meticulousness! First $C$ and $D$ are self-adjoint. Besides, $C D=D C$ as $N$ is normal. Then

$$
|N|^{2}=N^{*} N=(C-i D)(C+i D)=C^{2}+D^{2} \geq C^{2}
$$

as $D^{2} \geq 0$ because $D$ is self-adjoint. By Theorem 1.1, we get $|N| \geq|C|$. Hence, by Proposition 1.2

$$
-|N| \leq C \leq|N|
$$

and so

$$
|N|-C \geq 0 \text { and }|N|+C \geq 0 .
$$

Therefore, it makes sense to define their positive square roots. Consider (the selfadjoint!)

$$
A=\left(\frac{|N|+C}{2}\right)^{\frac{1}{2}} \text { and } B=\left(\frac{|N|-C}{2}\right)^{\frac{1}{2}} .
$$

Since $|N|$ commutes with $C$, it follows that $A$ commutes with $B$. Consequently, the operator $M:=A+i B$ is normal. Finally,

(1) If $D \geq 0$, then

$$
\begin{aligned}
M^{2} & =(A+i B)(A+i B) \\
& =A^{2}-B^{2}+i(A B+B A) \\
& =\frac{|N|+C}{2}-\frac{|N|-C}{2}+2 i\left(\frac{|N|^{2}-C^{2}}{4}\right)^{\frac{1}{2}} \\
& =C+i\left(D^{2}\right)^{\frac{1}{2}} \\
& =C+i D \\
& =N
\end{aligned}
$$

that is $M$ is a normal square root of $N$.

(2) A similar argument applies when $D \leq 0$. In this case,

$$
\left(M^{*}\right)^{2}=N,
$$

that is, $M^{*}$ is a normal square root of $N$. This marks the end of the proof.

This approach, besides its explicit construction, does apply for higher powers of the type $2^{n}$. In other language, the algorithm prescribed in the previous proof may be applied to deal with biquadratic equations or in general equations of the form $T^{2^{n}}=N$ where $n \in \mathbb{N}$. We have:

Corollary 3.2. Let $N=C+i D \in B(H)$ be normal with either $D \geq 0$ (or $D \leq 0$ ). Let $T \in B(H)$ be such that $T^{4}=N$. Then a normal 4 th root of $T$ is given by

$$
T=\left(\frac{|S|+\operatorname{Re} S}{2}\right)^{\frac{1}{2}}+i\left(\frac{|S|-\operatorname{Re} S}{2}\right)^{\frac{1}{2}}
$$

where

$$
S=\left(\frac{|N|+C}{2}\right)^{\frac{1}{2}}+i\left(\frac{|N|-C}{2}\right)^{\frac{1}{2}}
$$


Proof. Put $S=T^{2}$. Then $T^{4}=N$ becomes $S^{2}=N$. So, if e.g. $N$ is such that $\sigma(\operatorname{Im} N) \subset \mathbb{R}^{+}$, then $S$ is normal. Moreover, by Theorem 3.1, we know that

$$
S=\left(\frac{|N|+C}{2}\right)^{\frac{1}{2}}+i\left(\frac{|N|-C}{2}\right)^{\frac{1}{2}}
$$

and it is normal. Since clearly

$$
\operatorname{Im} S=\left(\frac{|N|-C}{2}\right)^{\frac{1}{2}} \geq 0,
$$

it follows that $T^{2}=S$ has a normal solution $T$ given by

$$
T=\left(\frac{|S|+\operatorname{Re} S}{2}\right)^{\frac{1}{2}}+i\left(\frac{|S|-\operatorname{Re} S}{2}\right)^{\frac{1}{2}} .
$$

Remark. By induction, we know how to find a root of order $2^{n}$ of normal operators very explicitly.

We finish with the case of general nth roots. First, the following result should be readily verified.

Lemma 3.3. Let $A \in B(H)$. If $k \in \mathbb{Z}$, then

$$
e^{i(A+2 k \pi I)}=e^{i A} .
$$

Theorem 3.4. Let $N \in B(H)$ be normal. Let $n \in \mathbb{N}$. Then $N$ has always an nth root which is also normal and given by

$$
N^{\frac{1}{n}}=|N|^{\frac{1}{n}} e^{i\left(\frac{A+2 k \pi I}{n}\right)}
$$

for some self-adjoint $A \in B(H)$ and where $k \in \mathbb{Z}$.

Proof. Since $N$ is normal, it may be expressed as (see e.g. [11])

$$
N=U P=P U
$$

where $U$ is unitary and $P=\sqrt{N^{*} N}=|N|$. Since $U$ is unitary, $U=e^{i A}$ for some self-adjoint $A \in B(H)$ (cf. Proposition 18.20 in [5]). Hence

$$
N=e^{i A} P=P e^{i A} \text {. }
$$

Set for $k \in \mathbb{Z}$

$$
M=P^{\frac{1}{n}} e^{i\left(\frac{A+2 k \pi I}{n}\right)}
$$

where $P^{\frac{1}{n}}$ is the unique positive nth root of $P$. Then $M$ is normal (cf. [6]) because it is a product of two commuting normal operators (in fact, a product of a positive operator and a unitary one). By the commutativity of the factors and simple results, we obtain

$$
M^{n}=\left(P^{\frac{1}{n}} e^{i\left(\frac{A+2 k \pi I}{n}\right)}\right)^{n}=P e^{i(A+2 k \pi I)}=P e^{i A}=N,
$$

as needed.

Remark. As is the case of many results on normal operators, the previous two theorems too are inspired by results about complex numbers. This corroborates and strengthens the ressemblance which is already known to readers. 


\section{REFERENCES}

[1] R. Bhatia, P. Rosenthal, How and why to solve the operator equation $A X-X B=Y$. Bull. London Math. Soc., 29/1 (1997) 1-21.

[2] J. B. Conway, B. B. Morrel, Roots and logarithms of bounded operators on Hilbert space, J. Funct. Anal., 70/1 (1987) 171-193.

[3] M. R. Embry, Similarities Involving Normal Operators on Hilbert Space, Pacific J. Math., 35 (1970) 331-336.

[4] S. Kurepa, On $n$-th roots of normal operators, Math. Z., 78 (1962) 285-292.

[5] R. Meise, D. Vogt, Introduction to Functional Analysis, Oxford G.T.M. 2, Oxford University Press 1997.

[6] M. H. Mortad, An Operator Theory Problem Book, World Scientific Publishing Co., (to appear in 2018).

[7] C. R. Putnam, On Square Roots of Normal Operators, Proc. Amer. Math. Soc., 8 (1957) 768-769.

[8] C. R. Putnam, On Square Roots and Logarithms of Self-adjoint Operators, Proc. Glasgow Math. Assoc., 4 (1958) 1-2.

[9] H. Radjavi, P. Rosenthal, On Roots of Normal Operators, J. Math. Anal. Appl., 34 (1971) 653-664.

[10] M. Rosenblum, On the Operator Equation $B X-X A=Q$, Duke Math. J., 23 (1956) 263-269.

[11] W. Rudin, Functional Analysis, McGraw-Hill Book Co., Second edition, International Series in Pure and Applied Mathematics, McGraw-Hill, Inc., New York, 1991.

[12] J. G. Stampfli, Roots of Scalar Operators, Proc. Amer. Math. Soc., 13/5 (1962) 796-798.

Department of Mathematics, University of Oran 1, Ahmed Ben Bella, B.P. 1524, El Menouar, Oran 31000, Algeria.

Mailing address:

Pr Mohammed Hichem Mortad

BP 7085 Seddikia Oran

31013

Algeria

E-mail address: mhmortad@gmail.com, mortad@univ-oran.dz. 